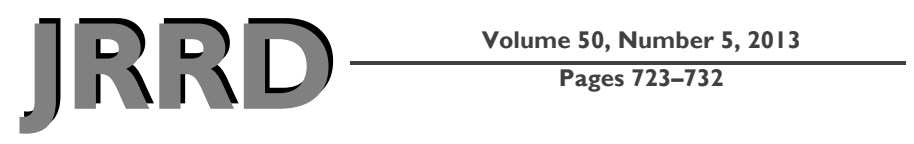

\title{
Comparison of mechanical properties of silicone and PVC (polyvinylchloride) cosmetic gloves for articulating hand prostheses
}

\author{
Gerwin Smit, MSc; ${ }^{*}$ Dick H. Plettenburg, MSc, PhD \\ Department of Biomechanical Engineering, Delft Institute of Prosthetics and Orthotics, Delft University of Technology, \\ Delft, the Netherlands
}

\begin{abstract}
Current articulating electric and body-powered hands have a lower pinch force $(15-34 \mathrm{~N})$ than electric hands with stiff fingers $(55-100 \mathrm{~N})$. The cosmetic glove, which covers a hand prosthesis, negatively affects the mechanical efficiency of a prosthesis. The goal of this study is to mechanically compare polyvinylchloride (PVC) and silicone cosmetic gloves and quantify the stiffness of the finger joints, the required actuation energy, and the energy dissipation during joint articulation. Six cosmetic gloves, identical in size but made from different materials, were mechanically tested: three PVC and three silicone. The silicone gloves required less work and dissipated less energy during flexing. They also had a lower joint stiffness and required a lower maximum joint torque. Based on energy requirements, joint stiffness, and required joint torque, the tested silicone glove is most suitable for application on an articulating hand prosthesis.
\end{abstract}

Key words: cosmetic glove, efficiency, energy, hand prosthesis, hysteresis, PVC glove, silicone glove, stiffness, testing of prosthetic and orthotic components, upper-limb prosthetics.

\section{INTRODUCTION}

\section{Problems with Current Hand Prostheses}

Many hand prosthesis users are dissatisfied with various aspects of their prosthesis [1-2]. Rejection rates of hand prostheses are high $(20 \%-40 \%)$ [3]. Prostheses should meet the basic user demands, which can be summarized by cosmesis, comfort, and control [4]. In practice, however, hand prostheses do not meet all demands simul- taneously. Body-powered hand prostheses require that the user deliver an uncomfortably high activation force $(60$ $130 \mathrm{~N}$ ) to produce only a small pinch force of $15 \mathrm{~N}$ [5-6]. Also, electric hand prostheses with articulating fingers produce a relatively low pinch force of 15 to $34 \mathrm{~N}$ [7-9]. The cosmetic glove counteracts hand closing, therefore reducing the pinch force in articulating electric hands and in voluntary-closing body-powered hands $[5,10]$.

\section{Cosmetic Gloves}

The main function of the cosmetic glove is to cover the hand mechanism and give the hand prosthesis a natural and cosmetically pleasing appearance. As an additional benefit, the glove protects the mechanism against moisture and dirt. Currently, two types of cosmetic gloves are available: polyvinylchloride (PVC) and silicone [11].

The PVC glove is relatively durable. It has a higher resistance to mechanical damage (e.g., puncture, tearing, abrasion) than the silicone glove. However, it is also relatively stiff and gets easily stained [11-12]. The sensitivity to staining can be reduced by treating the PVC glove with a special surface coating, and plasticisers inside the PVC

\footnotetext{
Abbreviations: $\mathrm{MCP}=$ metacarpophalangeal, $\mathrm{PIP}=$ proximal interphalangeal, $\mathrm{PVC}=$ polyvinylchloride.

*Address all correspondence to Gerwin Smit, MSc; Delft Institute of Prosthetics and Orthotics, Department of Biomechanical Engineering, Delft University of Technology, Mekelweg 2, 2628 CD, Delft, the Netherlands; +31152781688. Email: g.smit@tudelft.nl
}

http://dx.doi.org/10.1682/JRRD.2011.12.0238 
keep the glove flexible. However, in the longer term (e.g., long storage), the plasticisers migrate out of the material. The PVC will degrade and become stiff and brittle.

The silicone glove is more flexible. It is less susceptible to light radiation (visible and ultraviolet) and heat. However, it gets mechanically damaged easily and is less durable than the PVC glove $[11,13]$. Therefore, it needs to be replaced more often. Because the material and production costs of a silicone glove are higher, the replacement costs are also higher than that of a PVC glove. Silicone gloves have a higher surface friction [11], which is a benefit when holding objects but a drawback during dressing when the silicone sticks inside garment sleeves.

From a mechanical point of view, a cosmetic glove has undesirable properties. The glove imposes parasitic forces on the mechanism due to the stiffness of the glove material. The glove also dissipates energy because of the internal hysteresis of the glove material [10]. As a result, a body-powered prosthesis user has to deliver more energy. Also, electric prostheses require batteries with a larger capacity and motors that are more powerful, which may contribute to a higher device mass.

\section{Articulating Fingers}

Recently, electric hands with articulating fingers have become commercially available [7-8]. For body-powered prostheses, only one articulating hand is available, the Becker Imperial Hand (http://www.beckermechanicalhand.com). Hands with articulating fingers have mechanisms with multiple joints. Therefore, they have a higher energy dissipation than hands with stiff fingers. This results in either a lower pinch force or an increase in the required operating energy. The pinch force of the current articulating electric hands (15$35 \mathrm{~N})$ [7-8] is lower than the pinch force of the stiff-fingered electric prostheses $(\sim 100 \mathrm{~N})$ [14]. In order to increase pinch force and reduce energy demand of both body-powered and electrical hands, it is desirable to use a glove with a low stiffness and hysteresis. Although PVC and silicone gloves have been used for decades [11], very limited quantitative data exist on the stiffness and hysteresis of cosmetic gloves. Special gloves were designed for the i-limb (Touch Bionics; Livingston, United Kingdom) and the Bebionic (RSL Steeper; Leeds, England). However, no data were published on the mechanical properties of these gloves. Herder et al. measured the stiffness and hysteresis of a PVC cosmetic glove for movements of the thumb [10]. Currently, no data are available on the effect of cosmetic gloves on other joints. Also, no data are available on the stiffness and hysteresis of silicone cosmetic gloves. The Delft Institute of Prosthetics and Orthotics is currently developing a new prototype of a body-powered articulating hand. It would be desirable to use a standard cosmetic glove for this prototype, instead of a special glove designed for the i-limb or Bebionic because the purchase prices of these are considerably higher (3.5 to 6.5 times higher for the Bebionic and 5 to 10 times higher for the i-limb than standard gloves). Furthermore, standard-sized cosmetic gloves are available through multiple manufacturers. To select the right cosmetic glove, knowing its mechanical properties is necessary.

\section{Goal}

The goal of this study was to determine the contribution of a standard PVC and silicone cosmetic glove to the stiffness of the finger joints and to quantify the energy dissipation during articulation of the finger joints. These data enable the selection of the most efficient glove for a new prototype of an articulating hand. Furthermore, the data can be used in future development of prosthetic hands and cosmetic gloves.

\section{METHODS}

\section{Tested Gloves}

Six male midsize cosmetic gloves (size 7 3/4) were tested (Figure 1). This corresponds with the size of a small adult male or large adult female hand. Three were made of PVC and the other three of silicone (Table 1). The gloves were slush molded from the same hand model and therefore almost identical.

\section{Test Setup}

To test the fingers, an articulating finger frame was designed and built. The finger frame has one joint, which has two low-friction roller bearings. The finger frame can be flexed by pulling the flexor cable (diameter: $0.8 \mathrm{~mm}$ ) attached to a pulley (radius: $4.0 \mathrm{~mm}$ ). A dead mass of $1.0 \mathrm{~kg}$, attached to the extensor cable, exerts an extension torque of $43.2 \mathrm{Nmm}$ to the finger joint. The mass exerts a constant extension torque to the joint but does not add stiffness to the joint. The flexor cable is attached to a test bench, which measures and records the cable force and displacement. Figure 2 shows a schematic overview of the setup. The thumb of the glove was moved away to avoid collision with the fingers.

The metacarpophalangeal (MCP) joint of the thumb and the web space between the thumb and index finger were tested in a similar way. The thumb was pulled open 


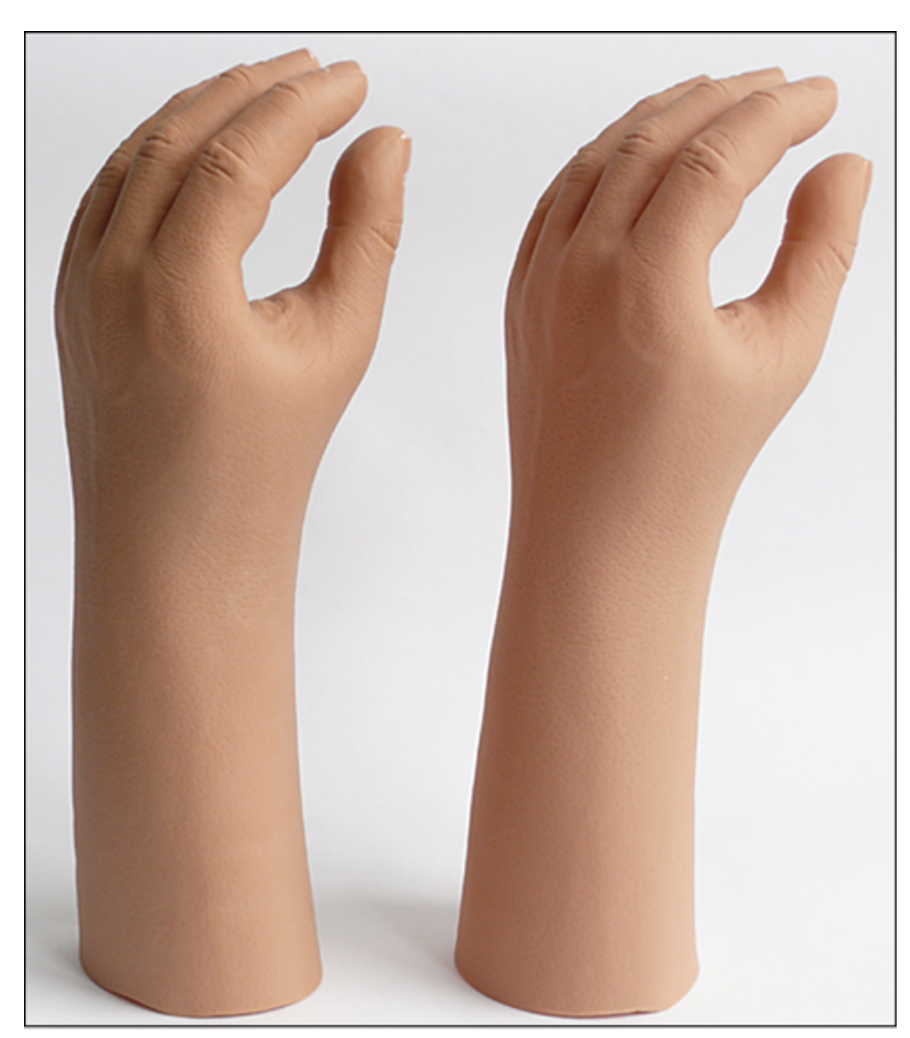

Figure 1.

Two tested cosmetic gloves: polyvinylchloride (PVC) (left) and silicone (right). Gloves were identical in size, shape, and texture. Only material differed. Fingers are slightly flexed in their neutral position.

from its neutral position, then closed again with the $1.0 \mathrm{~kg}$ counterweight (Figure 3).

\section{Test Protocol}

The thumb and index, middle, and ring fingers of the gloves were tested. For each finger, the MCP and proximal interphalangeal (PIP) joints were tested. For the thumb, the MCP joint was tested. For each test, the finger frame was placed inside the tested finger of the cosmetic glove. The joint of the frame was aligned with the tested joint location (MCP or PIP) of the cosmetic glove (Figure 2). Each finger joint was flexed $0.5 \pi \cdot \operatorname{rad}\left(90^{\circ}\right)$ and extended again. The thumb was extended $\pi / 3 \cdot \operatorname{rad}\left(60^{\circ}\right)$ from its neutral position and flexed again. Cable force and displacement were measured. The cable translations were used to calculate the joint angles, and the cable forces were used to calculate the joint torques. All tests were repeated three times to obtain average values. The tests were preceded by three unrecorded trials to avoid transient effects. The following parameters were measured or calculated from the measured data.

\section{Work and Hysteresis}

For each finger joint, the amount of work required to flex the joint from 0.1 to $0.5 \pi \cdot \mathrm{rad}$ was measured. The amount of dissipated energy, or hysteresis, was measured for the same interval. Instead of an interval of 0 to $0.5 \pi \cdot \mathrm{rad}$, the interval of 0.1 to $0.5 \pi \cdot \mathrm{rad}$ was considered. A counterweight of $1.0 \mathrm{~kg}$ was not heavy enough to fully extend the PVC glove fingers because the fingers are slightly flexed in the neutral position in which they are molded. For the thumb, an interval of 0 to $\pi / 3 \cdot \mathrm{rad}$ was considered.

Four empty runs were performed to determine the required work and hysteresis of the test setup. The setup required $18.9 \pm 0.04 \mathrm{Nmm}$ (mean \pm standard deviation) of work and had a hysteresis of $1.2 \pm 0.02 \mathrm{Nmm}$ for a joint rotation of $0.5 \pi \cdot \mathrm{rad}$. The system work and hysteresis were subtracted from the measured joint work and hysteresis.

\section{Maximum Joint Torque}

The maximum required joint torque $\left(\mathrm{M}_{\max }\right)$ was recorded for each joint.

\section{Average Joint Stiffness}

The average joint stiffness was calculated for each joint by making a linear least squares method fit to the data at an interval of 0.1 to $0.5 \pi \cdot \mathrm{rad}$ for the fingers and at an interval of 0 to $\pi / 3 \cdot \mathrm{rad}$ for the thumb.

\section{Glove or Skin Thickness}

As a result of the slush molding process, variations exist in glove thickness within a single glove and

Table 1.

Tested gloves.

\begin{tabular}{|c|c|c|c|c|c|}
\hline Glove & Material & Side & Size & Mass (g), Mean \pm SD & Brand \\
\hline$\overline{\mathrm{CG} 302 / \mathrm{E} 4}$ & PVC & $\mathrm{L}$ & $73 / 4$ & $338 \pm 23$ & RSL Steeper* \\
\hline SG302/E4 & Silicone & $\mathrm{L}$ & $73 / 4$ & $247 \pm 6$ & RSL Steeper* \\
\hline
\end{tabular}


(a)
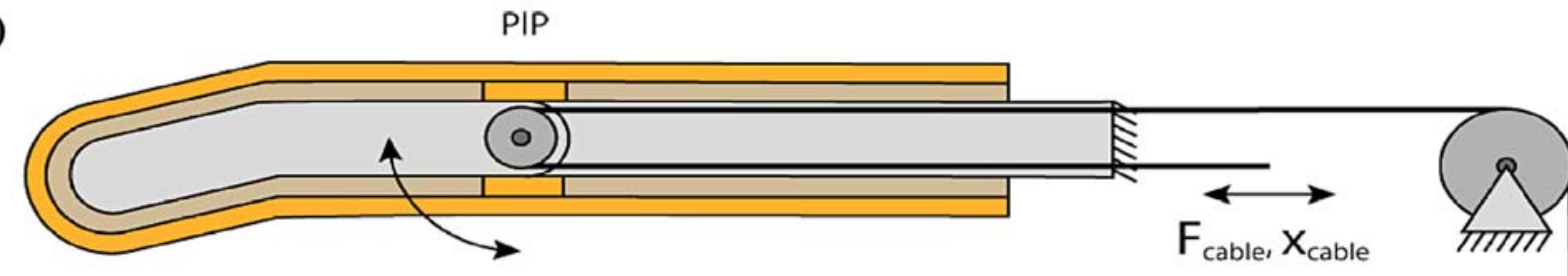

(b)

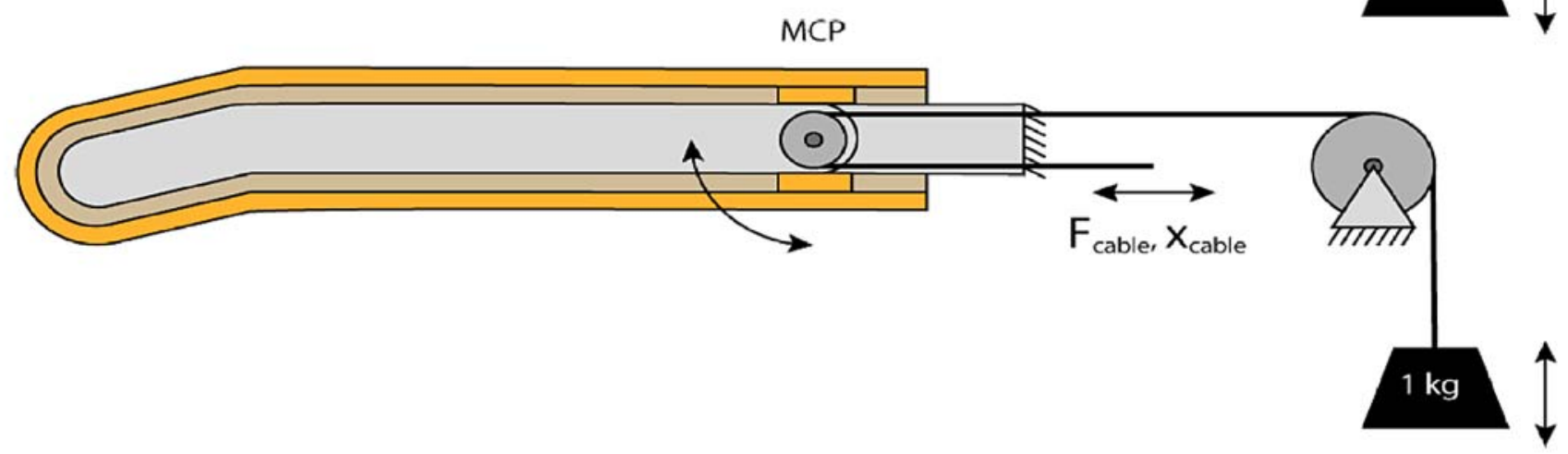

Figure 2.

Schematic overview of test setup configurations of (a) proximal interphalangeal (PIP) and (b) metacarpophalangeal (MCP) tests. Tested cosmetic glove is fitted over finger frame. Joint of finger frame is aligned with joint location of glove tested. Spaces between glove and frame are filled with soft foam (dark gray). $F=$ force, $X=$ displacement.

between different gloves. The glove thickness was measured for each tested joint for all gloves at the MCP and PIP joints. The finger of the glove was squeezed and the total thickness was measured using a micrometer caliper. The ratchet knob at the micrometer guarantees a constant caliper tip pressure for each measurement. Each joint was measured four times at different positions. The average of the measured thickness was divided by 2 to obtain the thickness of one glove layer.

\section{RESULTS}

\section{Angle-Torque Diagrams}

The angle-torque diagrams of the finger joints (Figure 4) show the measured torque for each joint angle between 0.1 and $0.5 \pi \cdot \mathrm{rad}$ for one cycle. For clarity, the data of one trial of one PVC and one silicone glove is shown. The data were representative for the other trials and gloves. Joint angles smaller than $0.1 \pi \cdot \mathrm{rad}$ were disregarded and are therefore not displayed. The diagrams show higher maximum joint torques for the PVC glove for each joint. The diagrams of the PVC glove run steeper, indicating a higher joint stiffness. They also enclose a larger area, which indicates a larger hysteresis for the PVC glove. This is similar for the thumb joint (Figure 5).

\section{Work and Hysteresis}

Table 2 shows the amounts of work and hysteresis that were measured for each joint. The work and hysteresis of the test setup are already subtracted from these values. The area between the upper line of a diagram and the $x$-axis represents the amount of work (Newton millimeter). The area enclosed by a diagram represents the hysteresis (Newton millimeter). The PVC glove required 1.8 to 3.8 times more work to flex the individual joints of the fingers. The PVC glove also dissipated 1.7 to 3.4 times more energy.

\section{Maximum Joint Torque}

The maximum required joint torque $\left(\mathrm{M}_{\max }\right)$ required to fully flex a joint was 2.2 to 4.2 times higher in the PVC gloves than the silicone gloves (Figure 6). 


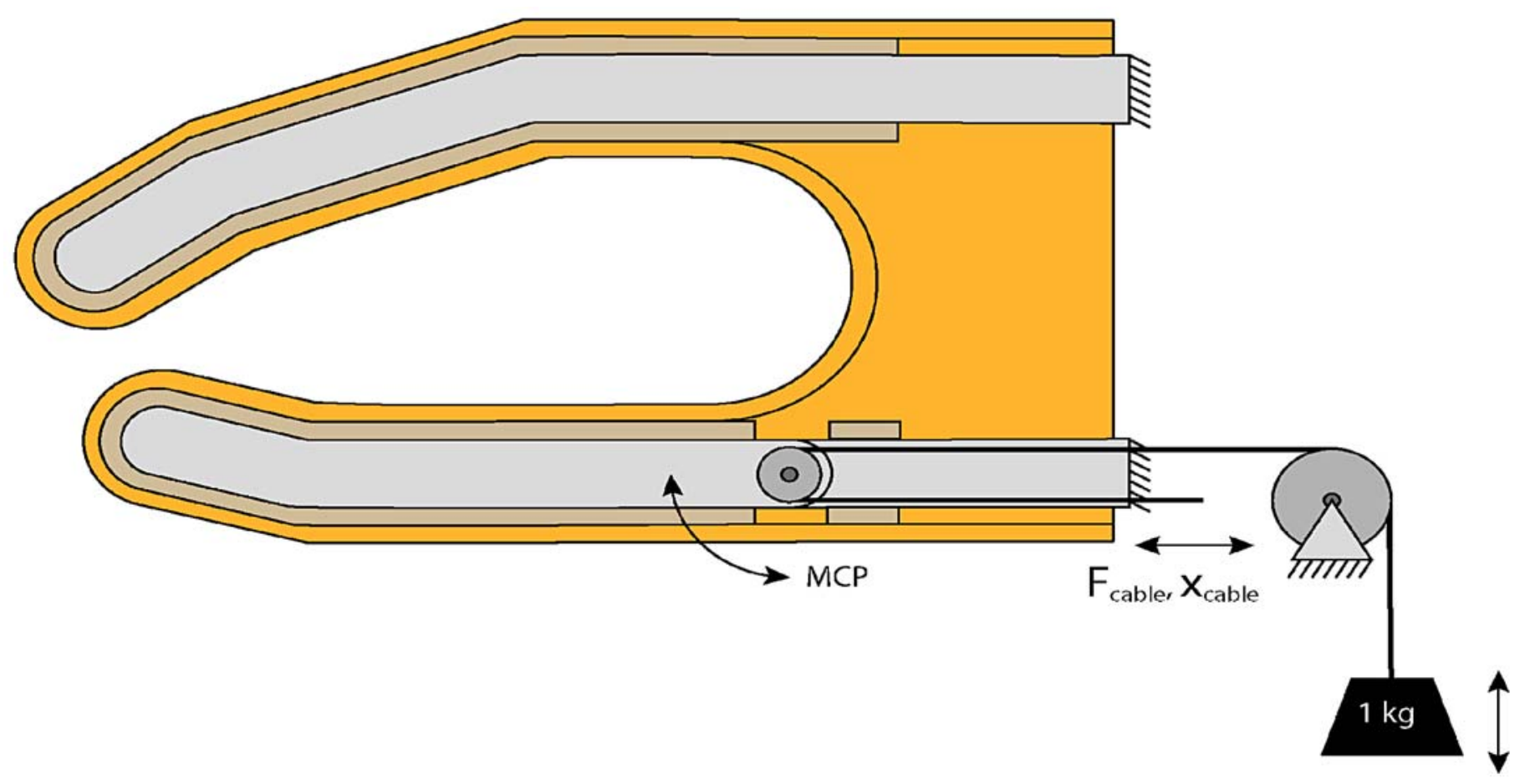

Figure 3.

Schematic overview of test setup configurations of metacarpophalangeal (MCP) joint of thumb and web space between thumb and fingers. Activation of cable opens thumb. Counterweight closes thumb when cable is released. Spaces between glove and frame are filled with soft foam (dark gray). $F=$ force, $X=$ displacement.

\section{Stiffness and Glove Thickness}

The stiffness of the PVC gloves was 2.5 to 4.5 times higher than the silicone gloves (Figure 7). Table 2 also shows the measured finger thickness. The fingers of the PVC gloves were 1.5 to 1.7 times thicker than the silicone gloves.

\section{DISCUSSION}

\section{Angle-Torque Diagrams}

The angle-torque diagrams of the fingers are only shown for 0.1 to $0.5 \pi \cdot \mathrm{rad}$ joint flexion. The finger joint did not fully extend when a PVC glove was applied. This is because the fingers of both gloves are already a little flexed in the neutral position in which they are molded, like in the real human hand. As a result, it required a negative torque to fully extend the finger. The counterweight of $1.0 \mathrm{~kg}$ was not heavy enough to fully extend the joint of a PVC glove. This was not a problem when a silicone glove was applied because it has a lower stiffness. It was undesirable to further increase the counter mass because this would result in a high load and friction inside the roller bearings. To make a fair comparison between both cosmetic gloves, it was decided to disregard the data between 0 to $0.1 \pi \cdot \operatorname{rad}$ for the finger joints.

\section{Work and Hysteresis}

The PVC gloves required considerably more work. They also dissipated considerably more energy. The sum of the required work of the MCP and PIP joints of the three tested fingers of the PVC glove was $119 \mathrm{Nmm}$. This seems relatively low when compared with closing a voluntary-closing Otto Bock hand $(1,710 \mathrm{Nmm})$ or Hosmer APRL hand $(1,058 \mathrm{Nmm})$ [5]. However, these hands all require very high activation forces. Non-hand-like gloveless prehensors, e.g., the TRS-GRIP (284 Nmm), require a much lower activation force because they have a relatively simple mechanism.

These devices are not meant to be covered. When we would add a cosmetic covering to such a device, this would considerably increase the total required work of the prehensor. Devices that have a more complex mechanism, 
(a)

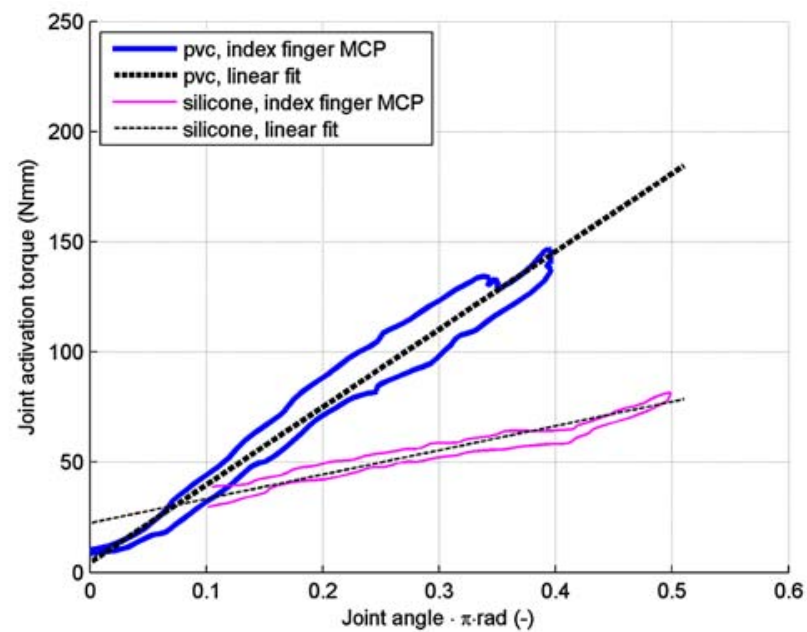

(c)

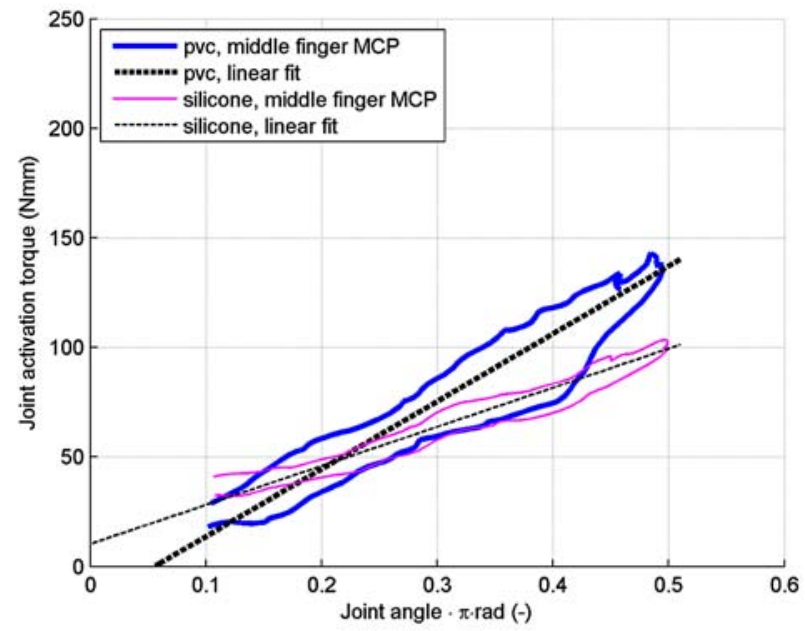

(e)

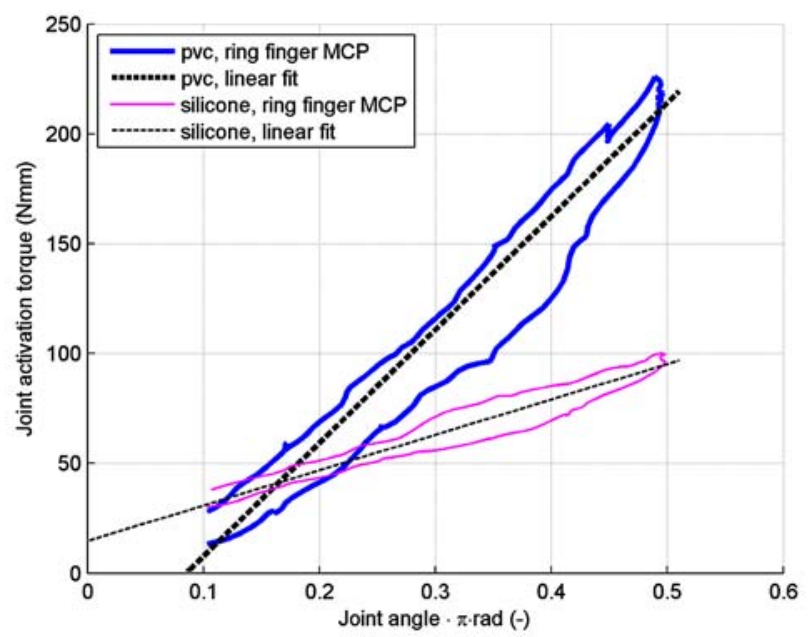

(b)

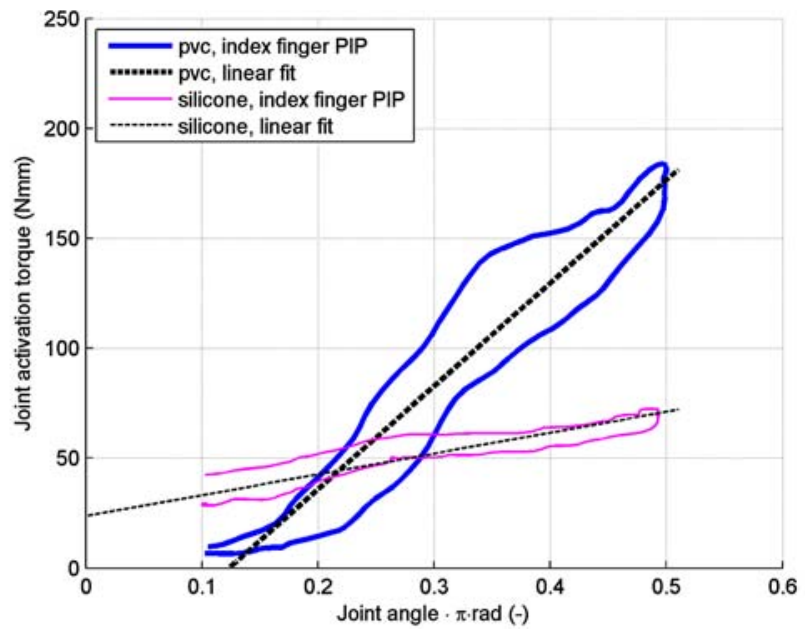

(d)

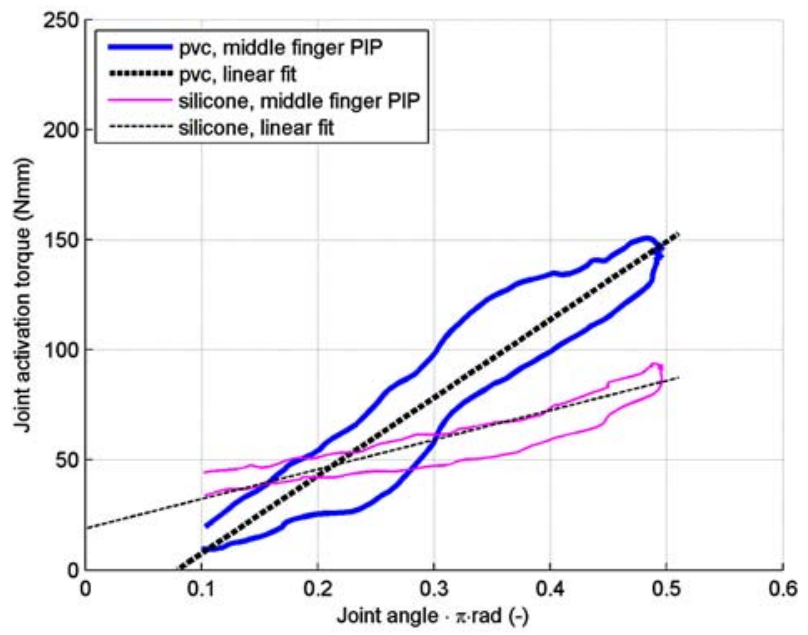

(f)

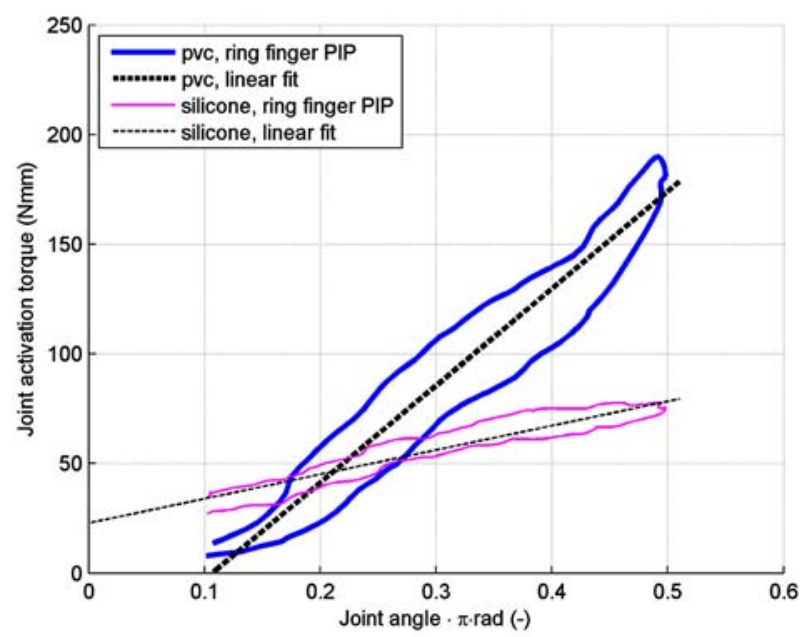

Figure 4.

Angle-torque diagrams of metacarpophalangeal (MCP) (left) and proximal interphalangeal (PIP) (right) joints of each finger: (a-b) index, (c-d) middle, and (e-f) ring. Thick lines represent polyvinylchloride (PVC) glove; thin lines represent silicone glove. For clarity, data of one trial of one PVC and one silicone glove is shown. Measured torque values include constant torque $(43.2 \mathrm{Nmm})$ produced by counter mass. 


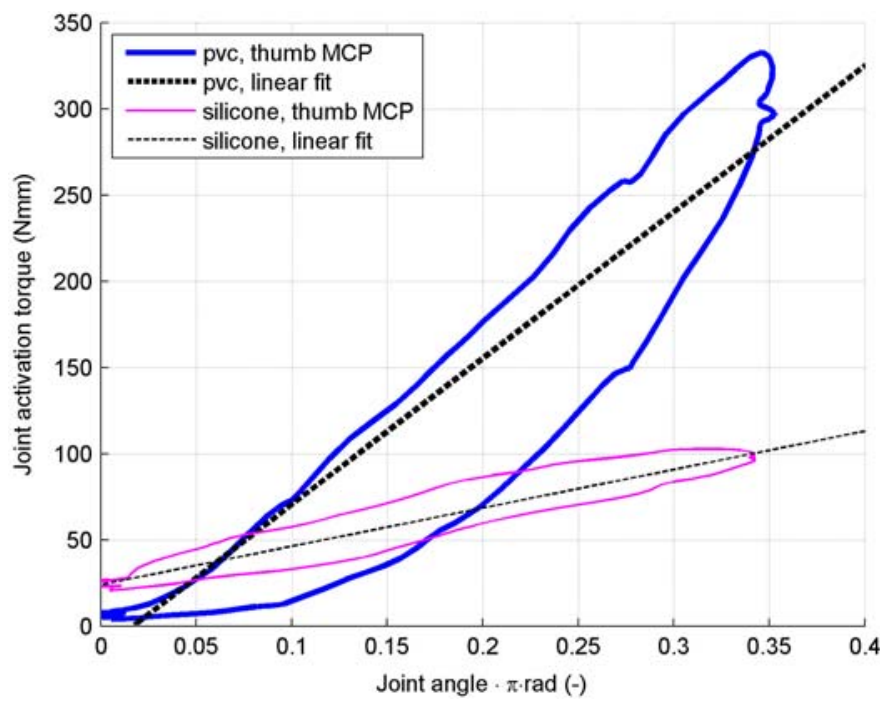

Figure 5.

Angle-torque diagram of metacarpophalangeal (MCP) thumb joint and web space. Note that scale for thumb differs from those of fingers. For clarity, data of one trial of one polyvinylchloride (PVC) and one silicone glove is shown. Measured torque values include constant torque (43.2 $\mathrm{Nmm}$ ) produced by counter mass.

with more joints and transmissions, require much more work than relatively simple mechanisms. Therefore, for the more complex devices, the work that is added by a cosmetic glove is relatively smaller. Also for these devices, however, the work added by the glove should be as low as possible, because the more complex mechanism itself requires so much more work from the user.

\section{Stiffness and Glove Thickness}

All the joints had a positive stiffness, which could be approximated well by a linear fit. The PVC gloves were much stiffer (2.5-4.5 times) than the silicone gloves. This can only partially be explained by the larger thickness of the PVC gloves because they were only 1.5 to 1.7 times thicker. The largest difference in stiffness $(350 \%)$ was measured for the PIP joint of the index finger, for which the thickness between the gloves only differed 67 percent. Therefore, it can be concluded that the main cause of the difference in glove stiffness is the difference in stiffness properties of the PVC and silicone glove material. For a minimal required input energy and a maximal pinch force, the stiffness should be as low as possible. To further reduce the drawbacks of glove stiffness, the glove stiffness can be compensated. This can be achieved by

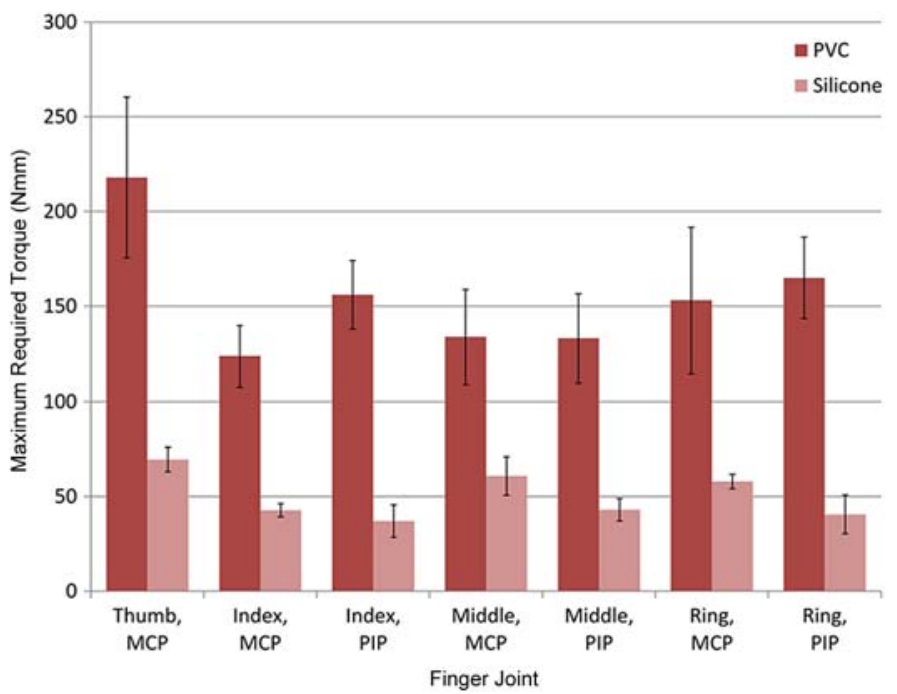

Figure 6.

Maximum joint torque to flex joint $0.5 \pi \cdot \mathrm{rad}\left(90^{\circ}\right)$. Maximum joint torque was up to 4.2 times higher in polyvinylchloride (PVC) glove. $\mathrm{MCP}=$ metacarpophalangeal, $\mathrm{PIP}=$ proximal interphalangeal .

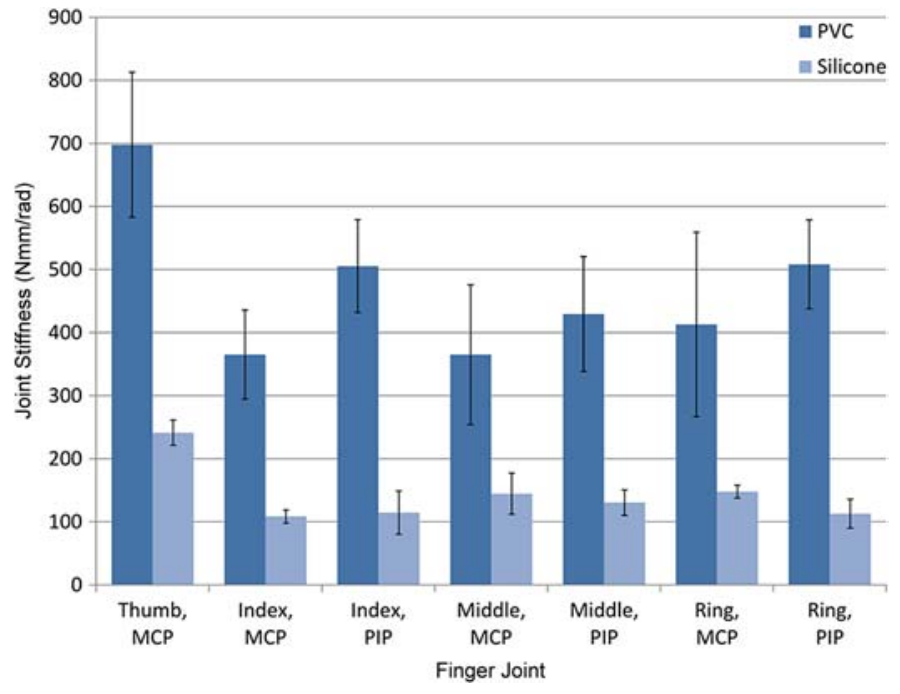

Figure 7.

Stiffness of different joints for polyvinylchloride (PVC) and silicone cosmetic gloves. Stiffness of PVC glove was up to 4.5 times higher than that of silicone glove. MCP = metacarpophalangeal, PIP = proximal interphalangeal.

using a stiffness compensation mechanism [15]. Because the glove has a linear positive stiffness, the compensation mechanism should have a linear negative stiffness. 
Table 2.

Work and hysteresis measured for every joint during $90^{\circ}$ flexion and extension of finger joints and $60^{\circ}$ of thumb joint and web space. Joint stiffness was calculated from measured data. Glove thickness was measured for each joint. Data presented as mean \pm standard deviation.

\begin{tabular}{|c|c|c|c|c|c|c|}
\hline Finger Joint & Material & $\begin{array}{c}\text { Work } \\
\text { (Nmm) }\end{array}$ & $\begin{array}{c}\text { Hysteresis } \\
\text { (Nmm) }\end{array}$ & $\begin{array}{l}M_{\max }{ }^{*} \\
(\mathrm{Nmm})\end{array}$ & $\begin{array}{c}\text { Stiffness } \\
\text { (Nmm/rad) }\end{array}$ & $\begin{array}{c}\text { Thickness } \\
\text { (mm) }\end{array}$ \\
\hline \multirow[t]{2}{*}{ Thumb MCP } & PVC & $27.5 \pm 7.7$ & $15.0 \pm 4.7$ & $218 \pm 42.4$ & $698 \pm 115$ & $1.6 \pm 0.2$ \\
\hline & Silicone & $13.9 \pm 0.5$ & $6.3 \pm 0.3$ & $69 \pm 6.5$ & $241 \pm 20$ & $0.9 \pm 0.1$ \\
\hline Index MCP & Silicone & $6.4 \pm 1.7$ & $3.1 \pm 1.8$ & $43 \pm 3.5$ & $108 \pm 11$ & $0.9 \pm 0.1$ \\
\hline Index PIP & PVC & $19.7 \pm 1.7$ & $12.5 \pm 2.1$ & $156 \pm 18.0$ & $505 \pm 74$ & $1.5 \pm 0.2$ \\
\hline Middle MCP & Silicone & $10.7 \pm 2.7$ & $6.5 \pm 3.0$ & $61 \pm 10.2$ & $145 \pm 33$ & $0.9 \pm 0.1$ \\
\hline \multirow[t]{2}{*}{ Middle PIP } & PVC & $17.3 \pm 2.1$ & $11.8 \pm 1.6$ & $133 \pm 23.4$ & $429 \pm 91$ & $1.5 \pm 0.2$ \\
\hline & Silicone & $5.4 \pm 0.6$ & $4.3 \pm 0.9$ & $43 \pm 5.9$ & $130 \pm 20$ & $0.9 \pm 0.1$ \\
\hline \multirow[t]{2}{*}{ Ring MCP } & PVC & $24.9 \pm 2.1$ & $11.0 \pm 1.6$ & $153 \pm 38.5$ & $413 \pm 146$ & $1.4 \pm 0.2$ \\
\hline & Silicone & $9.9 \pm 1.4$ & $4.6 \pm 1.1$ & $58 \pm 3.8$ & $148 \pm 10$ & $0.9 \pm 0.1$ \\
\hline
\end{tabular}

\section{Study Limitations and Strengths}

In this study, six gloves, three PVC and three silicone, by one manufacturer were tested. The outcomes may vary among other brands and types. However, it is unlikely that such variations will give a totally different outcome. The variations in the results among the joints and fingers were small, whereas the variations between the gloves were large. Both gloves were identical in size, shape, and texture. The only parameter that differed between the gloves was the material. This study clearly shows that the glove material has a considerable effect on the mechanical performance of a cosmetic glove and on the energy requirement of a prosthetic hand. The results are in line with the design choice of the designers of the i-limb and Bebionic. They used silicone when they designed special multilayered, reinforced gloves for these new hands.

\section{Implications for Nonarticulating Hands}

For the nonarticulating, or "stiff-fingered," hands, the largest glove deformation takes place in the web space between the thumb and fingers. The properties of this part of the glove are represented by the measurement of the thumb MCP joint and the web space. These results show that for the thumb joint, the stiffness is 2.9 times higher in the PVC glove than in the silicone glove. The required work is 2.0 times higher for the PVC glove than for the sili- cone glove. For nonarticulating hands, the tested silicone cosmetic gloves required less energy than the PVC gloves.

\section{Clinical Significance}

The outcomes of this study can help clinicians select the most suitable cosmetic glove for a patient. Based on the mechanical properties, the silicone gloves outperformed the PVC gloves. Using a silicone glove will increase the battery life for an externally powered prosthesis. For a body-powered prosthesis, it will result in a lower user effort and increased user comfort. However, when selecting a cosmetic glove, other properties should also be taken into account (e.g., durability, cosmetic appearance, cost, resistance to staining). The results of this study also give directions for manufacturers and researchers to develop improved cosmetic gloves and prosthetic hands.

\section{CONCLUSIONS}

Six identical standard cosmetic gloves of different materials were mechanically tested: three PVC and three silicone. The most efficient glove was selected to be used for a newly developed articulating hand prototype. Both types of glove showed a linear joint stiffness characteristic. The silicone gloves had the lowest joint stiffness for all 
joints. As a result, they required less energy for flexing the joint and dissipated less energy. Also, the joint torque to fully flex the joints was considerably lower than for the PVC gloves. Based on energy requirements, joint stiffness, and required joint torque, the silicone gloves had a higher mechanical efficiency than the PVC gloves. They dissipated less energy and they required a lower activation force. This will result in an increased battery life or user comfort depending on the type of prosthesis used.

\section{ACKNOWLEDGMENTS}

\author{
Author Contributions: \\ Study concept and design: G. Smit, D. H. Plettenburg. \\ Acquisition of data: G. Smit. \\ Analysis and interpretation of data: G. Smit. \\ Drafting of manuscript: G. Smit, D. H. Plettenburg. \\ Critical revision of manuscript for important intellectual content: \\ D. H. Plettenburg. \\ Study supervision: D. H. Plettenburg.
}

Financial Disclosures: The authors have declared that no competing interests exist.

Funding/Support: This material was unfunded at the time of manuscript preparation.

\section{REFERENCES}

1. Biddiss E, Beaton D, Chau T. Consumer design priorities for upper limb prosthetics. Disabil Rehabil Assist Technol. 2007;2(6):346-57. [PMID:19263565]

http://dx.doi.org/10.1080/17483100701714733

2. Biddiss E, Chau T. Upper-limb prosthetics: critical factors in device abandonment. Am J Phys Med Rehabil. 2007; 86(12):977-87. [PMID:18090439] http://dx.doi.org/10.1097/PHM.0b013e3181587f6c

3. Biddiss EA, Chau TT. Upper limb prosthesis use and abandonment: a survey of the last 25 years. Prosthet Orthot Int. 2007;31(3):236-57. [PMID:17979010] http://dx.doi.org/10.1080/03093640600994581

4. Plettenburg DH. Basic requirements for upper extremity prostheses: The WILMER approach. In: Proceedings of the 20th Annual International Conference of IEEE Engineering in Medicine and Biology; 1998 Oct 28-Nov 1; Hong Kong, China. p. 2276-81.

5. Smit G, Plettenburg DH. Efficiency of voluntary closing hand and hook prostheses. Prosthet Orthot Int. 2010;34(4): 411-27. [PMID:20849359] http://dx.doi.org/10.3109/03093646.2010.486390

6. Smit G, Bongers RM, Van der Sluis CK, Plettenburg DH. Efficiency of voluntary opening hand and hook prosthetic devices: 24 years of development? J Rehabil Res Dev. 2012;
49(4):523-34. [PMID:22773256]

http://dx.doi.org/10.1682/JRRD.2011.07.0125

7. Bebionic [Internet]. Product brochure. Leeds (England): RSL Steeper; 2013 [cited 2011 Dec 12]. Available from: http://www.bebionic.com

8. Touch Bionics [Internet]. i-limb ultra data sheet. Mansfield (MA): Touch Bionics; 2013 [cited 2011 Dec 12]. Available from: http://www.touchbionics.com/products/active-prostheses/ i-limb-ultra

9. Van der Niet O, Reinders-Messelink HA, Bongers RM, Bouwsema H, Van der Sluis CK. The i-LIMB hand and the DMC plus hand compared: a case report. Prosthet Orthot Int. 2010;34(2):216-20. [PMID:20470060] http://dx.doi.org/10.3109/03093641003767207

10. Herder JL, Cool JC, Plettenburg DH. Methods for reducing energy dissipation in cosmetic gloves. J Rehabil Res Dev. 1998;35(2):201-9. [PMID:9651892]

11. Bilotto S. Upper extremity cosmetic gloves. Clin Prosthet Orthot. 1986;10(2):87-89.

12. Pylatiuk C, Schulz S, Döderlein L. Results of an Internet survey of myoelectric prosthetic hand users. Prosthet Orthot Int. 2007;31(4):362-70. [PMID:18050007] http://dx.doi.org/10.1080/03093640601061265

13. Davies EW, Douglas WB, Small AD. A cosmetic functional hand incorporating a silicone rubber cosmetic glove. Prosthet Orthot Int. 1977;1(2):89-93. [PMID:615299]

14. Otto Bock HealthCare [Internet]. Upper extremity prosthetics catalog. Minneapolis (MN): Otto Bock HealthCare; 2013 [cited 2011 Dec 12]. Available from:

http://www.ottobockus.com/Prosthetics/Upper-LimbsProsthetics

15. Herder JL. Design of spring force compensation systems. Mech Mach Theory. 1998;33(1-2):151-61. http://dx.doi.org/10.1016/S0094-114X(97)00027-X

Submitted for publication December 14, 2011. Accepted in revised form October 29, 2012.

This article and any supplemental material should be cited as follows:

Smit G, Plettenburg DH. Comparison of mechanical properties of silicone and PVC (polyvinylchloride) cosmetic gloves for articulating hand prostheses. J Rehabil Res Dev. 2013;50(5):723-32.

http://dx.doi.org/10.1682/JRRD.2011.12.0238

ResearcherID/ORCID: Gerwin Smit, MSc: B-99942012; Dick H. Plettenburg, MSc, PhD: C-1078-2012

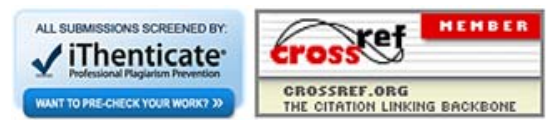


